\title{
Factors associated with massive fibrosis in silicosis
}

\author{
Tze Pin Ng, Shiu Lun Chan
}

\begin{abstract}
Data on 1432 patients with silicosis on a register in Hong Kong were analysed to examine the association of massive fibrosis with possible predisposing factors. Detailed occupational and clinical histories, clinical records, radiographic readings according to the International Standard Classification of Radiographs of Pneumoconioses, and environmental dust measurements from hygiene surveys were used to obtain information for several variables-namely, age at first exposure, relative dust exposure level, duration of exposure, smoking, previous recorded history of tuberculosis, and background profusion of small opacities. The most significant risk factors associated with massive fibrosis were high relative dust exposure level, a history of tuberculosis, and increased background profusion of small opacities.
\end{abstract}

Workers exposed to free silica develop nodular or stellate fibrotic lesions in the lungs that may coalesce into conglomerate or confluent lesions called massive fibrosis. Such complicated silicosis is usually associated with impaired lungs and disability, as well as premature death. Although the different degrees of fibrosis in simple and complicated disease are usually thought to be determined mainly by the amount of siliceous dust inhaled, ${ }^{1}$ this and other factors that may determine the occurrence of massive fibrosis have rarely been studied systematically in workers exposed to silica. In progressive massive fibrosis in coal workers by contrast the possible roles of coal rank, quartz, tuberculosis, other intercurrent infection, and immunological factors have been investigated extensively. ${ }^{1}$ In this study we have analysed data from 1432 patients in a population based silicosis register, to examine some factors that might determine or contribute to the development of massive fibrosis in workers with silicosis-namely, intensity and duration of dust exposure, age at first exposure, smoking, tuberculous infection, and background profusion of small opacities.

\section{Methods}

Silicosis has long been known to be an important occupational disease in Hong Kong. Voluntary notification of cases began in 1956, and workers with silicosis have been compensated for their disability since 1981, when a statutory scheme of compensation came into effect. Under this scheme all patients whose silicosis had been diagnosed by 31 December 1980 and who were residents of Hong Kong became eligible for ex gratia compensation from public funds. These individuals with confirmed silicosis were entered into a register (register I). Patients with silicosis diagnosed on or after 1 January 1981 were compensated under a different scheme, out of funds raised by levies from the industry at risk, and entered into a separate register (register II).

The study subjects were a total of 1487 workers with silicosis who were entered into register I. All patients with silicosis were seen in one pneumoconiosis clinic in the outpatient chest service for routine medicolegal documentation. This consisted of systematic and detailed histories, including occupational exposures and smoking habits as well as recorded treatment for pulmonary tuberculosis. Nearly all the patients had been registered informally at the pneumoconiosis clinic since 1974 (before that they were also registered as tuberculosis or silicosis patients receiving treatment from the chest service). For most of the patients who had received treatment for tuberculosis the diagnosis had been confirmed bacteriologically $(69 \%)$; the rest had the diagnosis made on radiological grounds $(31 \%)$. The diagnosis of silicosis was confirmed on the basis of occupational, clinical, and radiological evidence by a pneumoconiosis medical board. The radiological profusion of silicosis opacities was recorded by one consultant chest physician (who is also the medical board chairman) according to the International Standard Classification of Pneumoconioses, 1980 . $^{2}$ By definition, simple silicosis in this context included both rounded and irregular small opacities-that is both "classical" silicosis and "mixed dust fibrosis." A smoker was coded as either "ever smoker" or "never smoker."

The information on the jobs held during the patient's lifetime work experience was used to assign a relative level of silica dust exposure, weighted by the duration of each job. Most patients (over $90 \%$ ) had held jobs in one or more of three main categories of dusty work-namely, rock cutting and drilling, rock crushing, and unskilled general labour in the construction and quarry industries, nearly all being in the construction industry. The dust exposure levels and risks of silicosis in these industries have been described. ${ }^{3-5}$ Current environmental data (table 1) were used as guides in assigning relative dust exposure level ratings for broad occupational groups. Work processes and levels of dust control in the past in quarries and on construction sites were virtually the same, and have remained largely unchanged in the con- 
Table 1 Sample hygiene survey measurements of current average respirable dust concentrations and approximate exposure level rating by broad exposure groups ${ }^{\star}$

\begin{tabular}{lll}
\hline $\begin{array}{l}\text { Principal work processes } \dagger \\
\text { by industry }\end{array}$ & $\mathrm{mg} / \mathrm{m}^{3}$ & Rating \\
\hline Drilling & & \\
$\quad$ Quarries & $2 \cdot 09 \ddagger$ & \\
$\quad$ Construction & 3.24 & 3 \\
Crushing & $0.97 \ddagger$ & \\
$\quad$ Quarries & 2.98 & 2 \\
$\quad$ Construction & 1.08 & 1 \\
General labouring & Construction & \\
\hline
\end{tabular}

*All results refer to time weighted average concentration of respirable dust determined from a total of 334 personal samples.

†All other occupations were assigned exposure level ratings of 1 .

†Historical data (based on particle counts) indicate likely higher levels of dust exposure in the past.

struction industry. Historical data based on particle counts indicate that the past levels of dust exposure in quarries were considerably higher in the early 1950s than in recent decades. Within wide limits therefore past exposure in the quarries is likely to have approximated to current gravimetric estimates for exposure on construction sites rather than in quarries. Thus on the basis of estimates of dust exposure derived from the construction industry, for a rough approximation, workers concerned with drilling operations were assigned a dust exposure level rating of 3, those concerned with rock crushing 2 , and general labourers and all others 1. As workers commonly held multiple jobs, a lifetime relative dust exposure level for all jobs ever held, weighted by duration of employment in each job, was calculated for each person, who was then classified on the basis of groups with low $(<1 \cdot 0)$, intermediate $(1 \cdot 0-1 \cdot 9)$, and high $(\geqslant 2 \cdot 0)$ relative exposure levels.

Statistical analyses were performed as for cross sectional data, with cross tabulation and stratifications of categorical variables. Significance testing was by $\chi^{2}$ analysis and the Mantel-Haenszel procedures to estimate relative risks while possible confounding by multiple variables was controlled for. The analyses were performed with SAS software on microcomputers. ${ }^{6}$

\section{Results}

Data were available for analysis on 1432 patients after exclusion of 55 subjects with missing data (because information on some of the variables was incomplete). Fifty one percent of the patients with silicosis were classified as having large opacities of all sizes. The prevalence of large opacities was related to several factors-namely, associated profusion of small opacities, relative level of dust exposure, and recorded history of tuberculosis (table 2). The prevalence of large opacities appeared to be somewhat lower in those aged less than 40 years and exposed for less than 10 years, but this was not statistically significant at the $5 \%$ level. Smoking was not related significantly to the presence of large opacities.

The profusion of small opacities in subjects with simple silicosis was strongly associated with relative dust exposure level, duration of exposure, and tuberculosis. Tuberculosis was not associated with relative dust exposure level $\left(p=0.54, \chi^{2}\right.$ test $)$. The estimated relative risks of progressive massive fibrosis, when simultan-

Table 2 Numbers and proportions of patients with silicosis with and without large opacities by selected characteristics

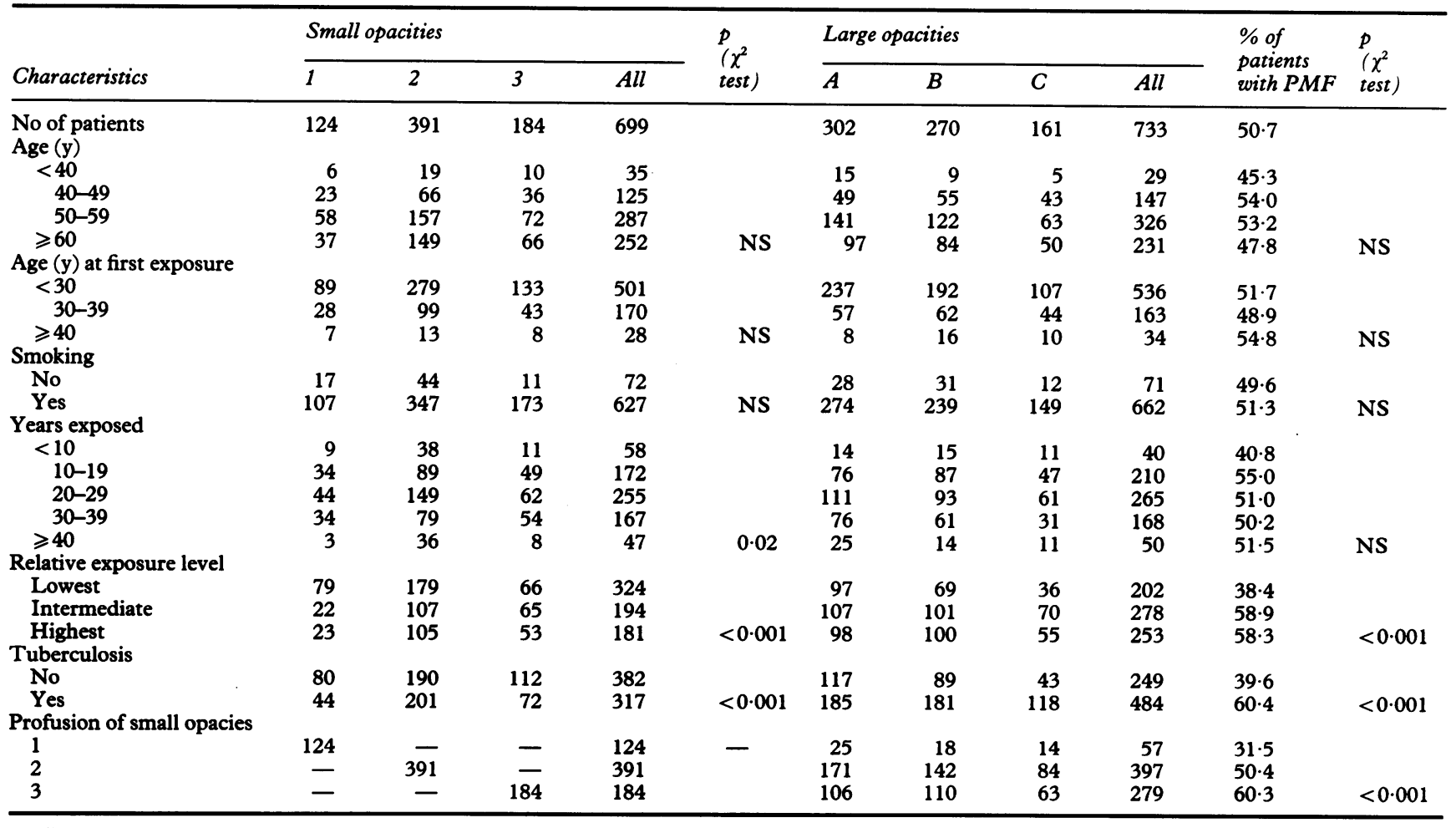

PMF—progressive massive fibrosis. 
Table 3 Relative risks of large opacities associated with selected variables

\begin{tabular}{ll}
\hline & $\begin{array}{l}\text { Relative risk } \\
(95 \% \text { confidence interval })^{\star}\end{array}$ \\
\hline $\begin{array}{l}\text { Tuberculosis } \\
\text { Profusion of small opacities }\end{array}$ & $1.52(1.37-1.69)$ \\
$\quad$ Category 1 & 1.00 \\
$\quad$ Category 2 & $1.42(1.17-1.73)$ \\
$\quad$ Category 3 & $1.64(1.36-1.98)$ \\
Relative exposure level & 1.00 \\
$\quad$ Lowest & $1.44(1.27-1.64)$ \\
$\quad$ Highest & $1.41(1.24-1.61)$ \\
\hline
\end{tabular}

^Relative risk for each variable was adjusted for possible confounding by all other variables in the table.

eous adjustments were made for the potentially confounding influences of relative dust exposure, profusion of small opacities, and tuberculosis, are shown in table 3. A significantly increased relative risk of massive fibrosis was associated with higher levels (intermediate and highest) of dust exposure, greater profusion (categories 2 and 3 ) of small opacities, and a history of tuberculosis, suggesting that each of these factors was independently and significantly associated with progressive massive fibrosis. Similar results were obtained when an additional adjustment was made for duration of exposure.

\section{Discussion}

The association of a category 2 or 3 background profusion of small opacities with progressive massive fibrosis appears to be consistent with the accepted histopathological view that massive fibrosis is formed by the conglomeration of individual fibrotic nodules (as in classical silicosis) or the confluence of irregular fibrotic lesions (as in mixed dust fibrosis). Studies of coal miners have also clearly shown that an increasing attack rate of progressive massive fibrosis is directly related to an increasing background profusion of small opacities. ${ }^{7}$ The same studies, however, have also indicated that, although massive fibrosis usually develops on a background of simple pneumoconiosis, some coal miners who develop it have no evidence of coal workers' pneumoconiosis. No such cases were identified in our population of patients with silicosis, but the possibility is not excluded. Physicians might be reluctant to ascribe large opacities to silicotic massive fibrosis alone in the absence of widespread uniform small opacities elsewhere in the lungs. A differential diagnosis of tuberculosis would usually be favoured in these circumstances, because of the high local prevalance of the infection. These patients may therefore be selected out of the study population.

Tuberculosis showed a relatively strong association with massive fibrosis in this study. The interpretation of this association, however, is difficult. The first problem is diagnostic accuracy, tuberculosis being difficult to distinguish radiologically from massive fibrosis. Bias could be introduced if the opacities in silicotuberculosis were diagnosed as massive fibrosis, or if cases of massive fibrosis were incorrectly diagnosed as tuberculosis, and thus not included in the register. Secondly, the available information in this study, which was based on records of cases of silicosis made at single points in time, did not allow interpretation of chronological events. Tuberculosis and massive fibrosis were frequent complications in these patients with silicosis, and indeed in many cases they were first diagnosed concurrently. This was partly due to the fact that exposed workers in the industry did not have periodic chest radiographic screening for silicosis, so most patients presented only when they had symptoms, by which time their disease was fairly advanced. This also explains the high proportion of patients with advanced silicosis in the register. In contrast to observations in Western countries, where tuberculosis usually occurs late in the disease, our impression (though we have no firm data to support this) is that tuberculosis tends to occur as an early complication of silicosis in our patients. In many cases it was difficult to establish which condition occurred first.

Although silicosis is generally held to predispose to the development of tuberculosis, some aspects of the interaction of the two conditions are not widely recognised. Animal experiments suggest that concomitant exposure to fibrogenic dust has an unfavourable influence on the course of induced tuberculosis, ${ }^{8-11}$ and that more fibrosis is produced by silicosis and tuberculosis combined than by either acting alone. ${ }^{12}{ }^{13}$ Although clinical evidence is limited, such a synergistic effect of silicosis and tuberculosis in producing a more proliferative fibrous reaction appears to be substantiated by general clinical impression. ${ }^{14}$ Moreover, tuberculosis may complicate simple silicosis as well as advanced disease. ${ }^{15}$ Looking out for recurrence of tuberculosis in our patients with silicotuberculosis is clearly important, particularly as most continue to be exposed after completing their course of treatment for tuberculosis (we have no evidence that recurrence is more likely in these patients, however, than those who are not further exposed). During the course of their silico-tuberculosis these same patients may also develop massive fibrosis. It has been postulated that tuberculous infection might contribute to the development of massive fibrosis in patients with silicosis. ${ }^{16}{ }^{17} \mathrm{Al}$ though our results support this, prospective studies are required.

Several pathogenetic processes are common to silicosis and tuberculosis and may be synergistic in producing more fibrosis (leading to massive fibrosis) or in enhancing susceptibility to mycobacterial infection or reactivation of latent infection. Quartz laden macrophages evoke cell death and fibrous proliferation as well as reduced resistance to tuberculosis. Lung fibrosis is common to the two diseases and irrespective of its cause may interfere with the clearance of dust laden alveolar macrophages or of infectious material from the lung. Obstruction to the lymphatic channels causes macrophages to accumulate in the interstitial tissues, thus predisposing them to local fibrous proliferation. ${ }^{1617}$ 
The present study also provides clear evidence of an association of massive fibrosis with high relative dust exposure level. The measure used was semiquantitative and admittedly crude, in addition to being subject to possible misclassification. Any such inaccuracies and errors in classification were, however, biased in favour of including some high exposure jobs in a lower relative exposure category. The relative risks of massive fibrosis were therefore likely to be underestimated for the higher relative exposure levels. A higher quartz content has been found in massive fibrosis tissue than in non-fibrotic control tissue. ${ }^{17}$ The evidence from this study is indirect for quartz dust, but supports the view that the quartz content of inhaled dust is an important primary cause of massive fibrosis.

Although a higher prevalence of massive fibrosis with increasing age and duration of exposure might be expected, this was not clearly shown in the present study. Among those with simple silicosis, however, the profusion of small opacities was significantly associated with duration of exposure. As the register included only those who were still alive on 31 December 1980, there is an inherent "survivor" bias in the register as it is likely to include all but the most rapidly fatal cases. The latter were more likely to be cases of massive fibrosis, and if they were more rapidly fatal in older patients this might explain why there is apparently no association between the prevalence of massive fibrosis and age or years of exposure. Moreover, the number of patients who were under 40 years or who had worked with silica for less than 10 years was relatively small. They were likely to be highly self selected because most of them were diagnosed because of symptomatic manifestations; a considerably larger number of cases of silicosis in "young" but symptom free individuals were likely to remain undiagnosed and therefore unregistered for many years. Many young patients with silicosis in the register were in fact unusual, having been exposed to extraordinarily high levels of silica. For instance, we have reported young patients with advanced silicosis who were exposed to extreme silica hazards from the use of silica flour. ${ }^{18}$ The relation may be further obscured if proportionately more silico-tuberculosis among relatively young individuals with silicosis of shorter duration was misclassified as massive fibrosis. Nevertheless, within the range of age and exposure duration of the study subjects, the effects of uniformly high exposure intensity and tuberculosis were likely to have been so large as to override many other factors.

Although some of these factors were interrelated-particularly relative dust exposure and profusion of small opacities and also profusion of small opactities and tuberculosis-the results of statistical adjustment suggested that, when this potential confounding was taken into account, each of these factors exerted an independent effect on the probability of the development of massive fibrosis in individuals with silicosis.

Thus in the lungs high quartz content would appear to be a primary factor in the pathogenesis of massive fibrosis in silicosis. Other factors, such as the coalescence of fibrotic nodules and tuberculous infection, could contribute through secondary "localising" processes. ${ }^{17}$ Immunological and other factors were not studied, but they could also have important roles in the pathogenesis of massive fibrosis in silicosis.

1 Parkes WR. Occupational lung disorders. 2nd ed. London: Butterworth, 1982:149, 192.

2 International Labour Office. Guidelines for the use of the ILO international classification of radiographs of pneumoconioses. Geneva: International Labour Office, 1980.

$3 \mathrm{Ng} \mathrm{TP}$, O'Kelly FJ. Silicosis prevalence in granite quarry and construction workers in Hong Kong. J Hong Kong Med Ass 1987;39:160-2.

$4 \mathrm{Ng} \mathrm{TP}$, Yeung KH, O'Kelly FJ. Silica hazard of caisson construction in Hong Kong. J Soc Occup Med 1987;37: $62-5$.

5 Ng TWK. Pneumoconiosis in Hong Kong: its epidemiology, compensation and control. MD thesis, University of Hong Kong, 1977:110-3.

6 SAS Institute. SAS procedures guide for personal computers. Version 6. Cary, North Carolina: SAS Institute Inc, 1985:373.

7 McLintock JS, Rae S, Jacobsen M. The attack rate of progrssive massive fibrosis in British coalminers. In: Walton WH, ed. Inhaled particles $I I I$, vol 2 . Old Woking: Unwin, 1971:933-52.

8 Gardner LW. Studies on experimental pneumokoniosis. V. Am Rev Tuberc 1929;20:833-75.

9 Policard A, Gernez-Rieux C, Tacquet A, Martin JC, Devulder B, Le Bouffant L. Influence of pulmonary dust load on the development of experimental infection by Mycobacterium kansasii. Nature 1967;216:177-8.

10 Ebina T, Takahashi Y, Hasuiki T. Effects of quartz powder on tubercle bacilli and phagocytes. Am Rev Respir Dis 1960;82:516-27.

11 Allison AC, D'Arcy Hart P. Potentiation by silica of the growth of Mycobacterium tuberculosis in macrophage cultures. Br J Exp Pathol 1968;49:465-76.

12 Gardner $L W$. Will the inhalation of siliceous dusts activate a partially healed focus of tuberculous infection? US Publ Health Rep 1930;45:282-8.

13 Policard A, Dufort A. Recherches experimentales sur les pneumoconio-tuberculoses. Ann Med Intern 1937;41: 183-209.

14 Bailey W, Brown $M$, Buechner $H$, Weill $H$, Ichinose $H$, Ziskind $\mathrm{M}$. Silico-mycobacterial disease in sandblasters. Am Rev Respir Dis 1974;110:115-25.

15 Westerholm P, Ahlmark A, Masing R, Segelberg I. Silicosis and risk of lung cancer or lung tuberculosis. Environ Res 1986;41:339-50.

16 Green GM. In defence of the lung. Am Rev Respir Dis 1970;102:691-703.

17 Leibowitz MC, Goldstein B. Some investigations into the nature and cause of massive fibrosis (MF) in the lungs of South African gold, coal and asbestos mine workers. Am J Ind Med 1987;12:129-43.

$18 \mathrm{Ng}$ TP, Allan WGL, Tsin TW, O'Kelly FJ. Silicosis in jade workers. Br J Ind Med 1985;42:761-4. 